\title{
Influence of the length parameter of an underground oil pipeline on the frequency of free oscillation
}

\author{
Vladimir Sokolov ${ }^{*}$, Igor` Razov ${ }^{1}$, and Andrey Dmitriev ${ }^{1}$ \\ ${ }^{1}$ Industrial University of Tyumen, Volodarskogo str.38, Tyumen, 625001, Russian Federation
}

\begin{abstract}
The problem of finding the natural frequencies of thin-walled underground oil pipelines is solved, based on the application of a semimomentless theory of cylindrical shells of medium bending, in which bending moments in the longitudinal direction are not taken into account in view of their smallness compared with moments acting in the transverse direction. The solution to this approach is a fourth-order homogeneous differential equation satisfying the boundary conditions of articulation at each end. This equation includes the parameters of the length, internal pressure, thinness of the pipeline, as well as the values of the coefficient of elastic resistance of the soil, the attached mass of the soil and the attached mass of the flowing oil. Based on the data obtained by the derived formulas, the frequency characteristics of large-diameter thin-walled underground oil pipelines are determined depending on the length of the element, as well as on the soil conditions. It has been established that the minimum frequencies are realized for shell modes of vibration with a length parameter of the pipeline section (the ratio of the length of the section to the radius) not exceeding 13. A formula is derived that allows one to determine the boundary between the use of the rod and shell theory for calculating pipelines for dynamic effects. Using the dynamic stability criterion, in which the frequency of natural oscillations vanishes, expressions are derived that allow one to determine the external critical pressure on the wall of the pipeline, which takes into account the length of the pipeline, as well as the number of half waves in the transverse and longitudinal directions, in which the pipeline goes into emergency condition.
\end{abstract}

\section{Introduction}

During operation, large-diameter trunk pipelines undergo various kinds of dynamic influences. Such an effect can be caused by seismic vibrations, or, for example, periodic vibrations caused by a passing train near a linear object from impacts of wheels against rail junctions.

The task of ensuring the reliability of the pipeline under dynamic conditions is to exclude resonance, that is, it is necessary to know the frequency of natural vibrations and

${ }^{*}$ Corresponding author: sokolovvg@tyuiu.ru 
forced vibrations of the system. Work on determining the dynamic characteristics of pipelines is reflected in the writings [1 - 13]. Most of the above works [1 - 7] are based on the rod theory, which does not take into account the deformation of the cross section of the pipe. Pipes with a diameter of more than $1000 \mathrm{~mm}$, which are used in the construction of modern oil pipelines, are difficult to characterize as a rod; therefore, it is not advisable to calculate these pipelines using methods based on the rod theory.

The foundations of the linear theory of small vibrations of thin-walled shells were laid by A. Lyav, however, the equations he derived did not get practical application, since they were too complicated, so a number of assumptions were introduced to simplify. The theory of closed cylindrical shells is suitable for practical use, from the variants of which the most complete theory of V. Flyge can be singled out, in which the equations of rotation inertia are neglected for the equations proposed by A. Lyav. For a closed cylindrical shell with articulations at ends, a solution is proposed for a system of three homogeneous differential equations of motion in displacements. This system of equations is solved using Fourier series, and the result is a cubic equation with respect to the square of the frequency of free vibrations:

$$
\alpha_{3} \omega^{6}+\alpha_{2} \omega^{4}+\alpha_{1} \omega^{2}+\alpha_{0}=0
$$

The solution also did not receive wide application because of complexity, therefore, for simplicity, from the equation of W. Fluge, the terms containing small factors with squares of the ratios of the shell thickness $h$ to the radius of the middle surface $R$ were discarded. Based on such simplifications by Kh.M. Mushtari, V.Z. Vlasov, as well as L.Kh. Donnel [14] a practically applicable equation of motion for a closed cylindrical shell is obtained, which is widely used at present.

This article raises the question of a new approach to the dynamic calculation of largediameter thin-walled underground oil pipelines, which is based on the semi-momentless theory of medium-bend shells. In this theory, bending moments in the longitudinal direction are neglected in view of their smallness in comparison with the moments acting in the transverse direction. The solution to this approach is a fourth-order homogeneous differential equation satisfying the boundary conditions of articulation at each end.

\section{Statement of the problem}

The design scheme (Fig. 1) is a closed cylindrical shell of length $L$, wall thickness $h$, and radius $R$, the internal working pressure $p_{0}$ acts on the shell wall, the external soil pressure $q_{s l}=H \gamma$, the reaction of elastic pressure response $q_{0}$, as well as the action of flowing fluid velocity $V$ and longitudinal compressive force $F$.

To take into account the hydrostatic pressure $q_{l}$ created by the flowing oil stream at a velocity $V$, the solution obtained by M.A. Ilgamov, A.S. Volmir is used:

$$
\begin{gathered}
q_{l}=\rho_{0} \Phi_{m n} \frac{R}{E h}\left(R^{2} \frac{\partial^{4} w}{\partial \theta^{2} \partial t^{2}}+V^{2} \frac{\partial^{4} w}{\partial \theta^{2} \partial \xi^{2}}\right) ; \\
\Phi_{m n}=\frac{I_{m}\left(\lambda_{0}\right)}{\lambda_{0} I_{m}^{\prime}\left(\lambda_{0}\right)} \text { is a paramet }
\end{gathered}
$$

where $\rho_{0}$ is the density of the liquid; $\quad \lambda_{0} I_{m}^{\prime}\left(\lambda_{0}\right)$ is a parameter depending on the wave numbers in the circumferential and longitudinal directions $(m, n)$ and is determined by the ratio of the Bessel function to its derivative depending on $\lambda_{0}=\frac{n \pi R}{L}, V$ is the fluid flow velocity, the product $\rho_{0} \cdot \Phi_{m n}$ is the added mass of the fluid. 


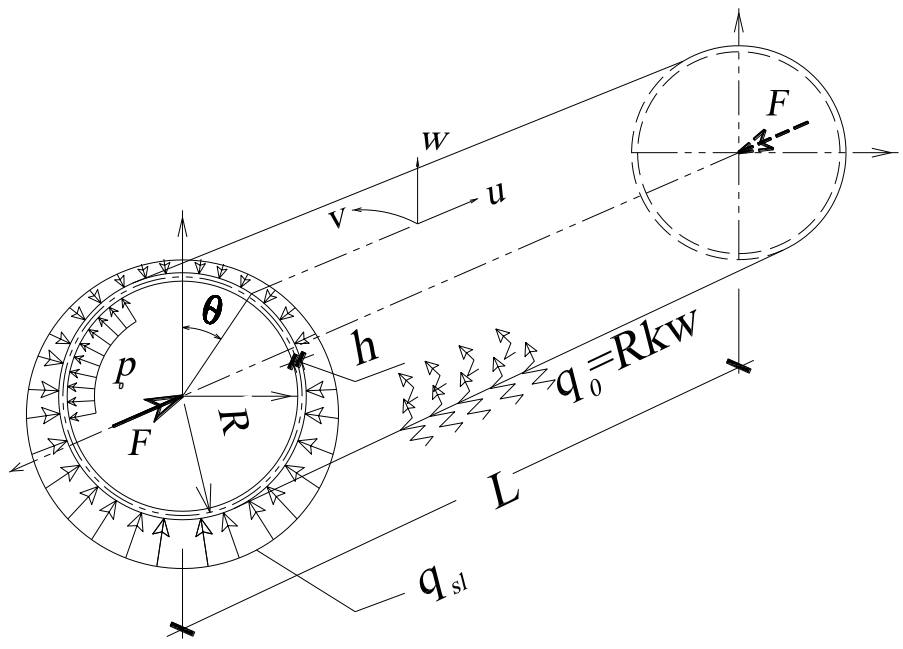

Fig. 1. The design scheme of the pipeline

To solve the problem of frequency characteristics of an large-diameter underground thin-walled oil pipeline, the force equation obtained in $[8,9]$ is used:

$$
\begin{aligned}
& \frac{\partial^{2} T_{1}}{\partial \xi^{2}}+\frac{\partial}{\partial \xi}\left(\tau \frac{\partial M_{2}}{\partial \theta}\right)-\frac{\partial^{2}}{\partial \theta^{2}}\left(\frac{R_{2}^{*}}{R_{1}^{*}} T_{1}\right)-\frac{1}{R^{2}} \frac{\partial^{2}}{\partial \theta^{2}}\left(R_{2}^{*} \frac{\partial^{2} M_{2}}{\partial \theta}\right)- \\
& -\frac{\partial}{\partial \theta}\left(\frac{1}{R_{2}^{*}} \frac{\partial M_{2}}{\partial \theta}\right)+R \frac{\partial X_{1}}{\partial \xi}-R \frac{\partial X_{2}}{\partial \theta}-\frac{\partial^{2}}{\partial \theta^{2}}\left(R_{2}^{*} X_{3}\right)=0
\end{aligned}
$$

the effect of a stationary fluid flow on the pipeline wall is taken into account in the normal component of the inertia forces $\mathrm{X} 3$ :

$$
\begin{aligned}
X_{3}= & -R h \rho \frac{\partial^{2} w}{\partial t^{2}}-\rho_{0} \Phi_{m n} \frac{R}{E h}\left(R^{2} \frac{\partial^{4} w}{\partial \theta^{2} \partial t^{2}}+V^{2} \frac{\partial^{4} w}{\partial \theta^{2} \partial \xi^{2}}\right)+p_{0}- \\
& -\gamma H\left(2-\alpha_{1} \cos \theta-\alpha_{2} \cos 2 \theta\right)-\kappa R w,
\end{aligned}
$$

Solving equation (3) using the assumptions of the semi-momentless theory of cylindrical shells after conversion, we obtain the differential equation of motion of the oil pipeline in displacements:

$$
\begin{gathered}
\frac{\partial^{3} u}{\partial \xi^{3}}+h_{v}^{2} \frac{\partial^{3}}{\partial \theta^{3}}\left(\frac{\partial^{2} \vartheta_{2}}{\theta}+\vartheta_{2}\right)+2 \frac{\partial^{2}}{\partial \theta^{2}}\left(\frac{\partial^{2} w}{\partial \xi^{2}} \varepsilon_{0}\right)-\frac{R}{E h} p_{0} \frac{\partial^{3} \vartheta_{2}}{\partial \theta^{3}}+\frac{R^{2} w \kappa \cdot \partial^{2} w}{E h \cdot \partial \theta^{2}}+ \\
+R \gamma H \frac{\partial^{3} \vartheta_{2}}{\partial \theta^{3}}\left(2-\alpha_{1} \cos \theta-2 \cos 2 \theta\right)+R \gamma H\left(\frac{\partial^{2} \vartheta_{2}}{\partial \theta^{2}} \alpha_{1} \sin \theta+\frac{\partial^{2} \vartheta_{2}}{\partial \theta^{2}} 2 \alpha_{2} \sin 2 \theta\right)+ \\
+R \gamma H\left(\frac{\partial \vartheta_{2}}{\partial \theta} \alpha_{1} \cos \theta-\frac{\partial \vartheta_{2}}{\partial \theta} 4 \alpha_{2} \cos 2 \theta\right)-\frac{R^{2} \rho}{E}\left(\frac{\partial^{3} u}{\partial \xi \partial t^{2}}-\frac{\partial^{3} v}{\partial \xi \partial t}-\frac{\partial^{3} w}{\partial \theta^{2} \partial t^{2}}\right)+ \\
+\rho_{0} \Phi_{m n} \frac{R}{E h}\left(R^{2} \frac{\partial^{4} w}{\partial \theta^{2} \partial t^{2}}+V^{2} \frac{\partial^{4} w}{\partial \theta^{2} \partial \xi^{2}}\right)=0
\end{gathered}
$$

where $\mathrm{u}, \mathrm{v}, \mathrm{w}$ are the components of the displacements of the middle surface of the shell, referred to the radius $R, \vartheta_{2}$ is the angle of rotation, $p_{0}$ is the internal pressure in the pipe, $\rho$ is the lateral pressure coefficient of the soil, $H$ is the thickness of the crimped layer, $\gamma$ is the volumetric weight of the soil, $E$ is modulus of elasticity of the pipe material, $R$ is 
radius the middle surface, $\quad h_{v}=\frac{h}{R \sqrt{12\left(1-v^{2}\right)}}$ is the parameter of the relative thickness of the shell, $\mu_{b j}$ is the added mass of soil per unit length of the pipeline, $\kappa$ is the coefficient of elastic resistance of the soil for the pipeline subjected to internal working pressure, presented in the form:

$$
\kappa=\frac{E_{0}}{R\left(1+v_{0}\right)}
$$

The resulting system of equations (4) contains four unknown functions of coordinates and time t: u, v, w and $\vartheta_{2}$. Based on the Fourier method (variable separation method), we represent a function $w(\xi, \theta, \mathrm{t})$ satisfying the condition of articulated support of the ends of the pipeline and periodicity along the circumferential coordinate $\theta$, in the form:

$$
w=\sum_{m} \cdot \sum_{n} b_{m n} \varphi(t) \sin \left(\overline{\lambda_{n}} \xi\right) \cos (m \theta)
$$

where

$$
\overline{\lambda_{n}}=\frac{n \pi R}{L}, m, n=1,2 \ldots .
$$

are the wave numbers in the circumferential and longitudinal directions.

The remaining components of the displacements and the angle of rotation $\vartheta_{2}$ are determined from the relations of the semi-momentless theory of shells:

$$
\begin{gathered}
u=-\sum_{m} \cdot \sum_{n} b_{m n} \frac{\overline{\lambda_{n}}}{m^{2}} \cos \left(\overline{\lambda_{n}} \xi\right) \cos (m \theta) ; \\
v=-\sum_{m} \cdot \sum_{n} b_{m n} \frac{1}{m} \sin \left(\overline{\lambda_{n}} \xi\right) \sin (m \theta) ; \\
\vartheta_{2}=-\sum_{m} \cdot \sum_{n} b_{m n} \frac{m^{2}-1}{m} \sin \left(\bar{\lambda}_{n} \xi\right) \sin (m \theta) .
\end{gathered}
$$

Given that the free vibrations of the shell carry out movement according to a harmonic law, we have:

$$
\varphi(t)=\sin \omega_{m n} t, \varphi^{\prime \prime}(t)=-\omega^{2} \sin \omega_{m n} t,
$$

where $\omega_{\mathrm{mn}}$ is the first frequency of free bending vibrations in shape, $\mathrm{m}, \mathrm{n}=1,2,3 \ldots$

Substituting (7)-(9) into equation (5) and equating the coefficients for the same trigonometric functions $\cos (\mathrm{m} \theta)$ for $\mathrm{m}, \mathrm{n}=1,2,3 \ldots$, we obtain an infinite system of homogeneous linear algebraic equations with respect to the unknown amplitude values $b_{n m}$ of the radial component moving $w$. The coefficients for the unknowns in these equations are denoted by $\alpha_{i j}$ :

$$
\begin{array}{ll}
\text { if } \mathrm{m}=1 & a_{1,1} b_{1}+a_{1,2} b_{2}+a_{1,3} b_{3}=0 ; \\
\text { if } \mathrm{m}=2 & a_{2,1} b_{1}+a_{2,2} b_{2}+a_{2,3} b_{3}+a_{2,4} b_{4}=0 ; \\
\text { if } \mathrm{m}=3 & a_{3,1} b_{1}+a_{3,2} b_{2}+a_{3,3} b_{3}+a_{3,4} b_{4}+a_{5,5} b_{5}=0 .
\end{array}
$$

The resulting system of linear homogeneous algebraic equations is written in the form:

$$
a_{m, m-2} b_{m-2, n}+a_{m, m-1} b_{m-1, n}+a_{m, m} b_{m, n}+a_{m, m+1} b_{m+1, n}+a_{m, m+2} b_{m+2, n}=0,
$$

where $\mathrm{m}=1,2,3 \ldots ; \mathrm{m} \pm 1>0 ; \mathrm{m} \pm 2>0$, and the coefficients $\alpha_{i j}$ are determined by the expression:

$$
\begin{gathered}
a_{m, m}=A_{m, n}-B_{m, n} \omega_{m n}^{2} ; a_{m, m \pm 1}=-\frac{m^{5}(m \pm 2)}{2} q_{s l}^{*} \alpha_{1} ; \\
a_{m, m \pm 2}=-\frac{m^{4}\left[(m \pm 2)^{2}-1\right]}{2} q_{s l}^{*} \alpha_{2}
\end{gathered}
$$




$$
\begin{gathered}
A_{m, n}=\lambda_{n}^{4}+m^{4}\left(m^{2}-1\right)\left(m^{2}-1+p^{*}-2 q_{s l}^{*}\right)+\kappa^{*} m^{4}-\lambda_{n}^{4} m^{4} P / n^{2} \\
B_{m, n}=\rho^{*} R h\left(\lambda^{2} h_{v}+m^{2}+m^{4}\right)+\mu_{b j}^{*} m^{4}+\rho_{0} \Phi_{m n} R^{2} m^{4}
\end{gathered}
$$

where $p^{*}=p_{0} \frac{R}{E h \cdot h_{v}^{2}} ; \rho^{*}=\rho_{0} \frac{R}{E h \cdot h_{v}^{2}} ; \kappa^{*}=\frac{R^{2} \kappa}{E h \cdot h_{v}^{2}} ; q_{s l}^{*}=\frac{\gamma H R}{E h \cdot h_{v}^{2}} ; \lambda_{n}=\frac{n \pi R}{L \sqrt{h_{v}}} ;$

$P=\frac{F}{F_{E}}$; where $\mathrm{F}$ is the longitudinal force; $F_{E}$ is Euler's force; $I=\pi R^{3} h$ is the moment of the section inertia.

The coefficients of this system of equations (10) are dimensionless at an internal working pressure $p_{0}$ in $\mathrm{MPa}$, an elastic resistance coefficient $\kappa$ in $\mathrm{kN} / \mathrm{m}^{3}$, and a shell material density $\rho_{0}$ in $\left(\mathrm{kN} \cdot \mathrm{s}^{2}\right) / \mathrm{m}^{4}$.

The task of determining the frequencies and forms of natural vibrations of an underground rectilinear thin-walled section of the pipeline is reduced to determining the eigenvalues of the matrix. To solve it, the determinant is given in matrix form (1):

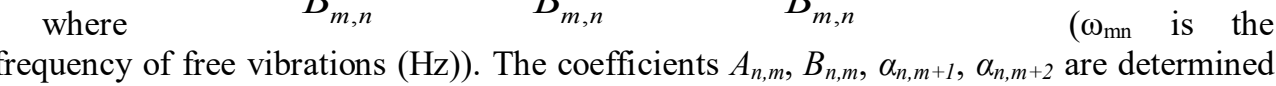

$$
\left|\begin{array}{cccccc}
d_{11}-\lambda & d_{12} & d_{13} & d_{14} & \ldots . . & d_{1 n} \\
d_{21} & d_{22}-\lambda & d_{23} & d_{24} & \ldots . . & d_{2 n} \\
d_{31} & d_{32} & d_{33}-\lambda & d_{34} & \ldots . . & d_{3 n} \\
d_{41} & d_{42} & d_{43} & d_{44}-\lambda & \ldots . . & d_{4 n} \\
\ldots . & \ldots \ldots & \ldots . . & \ldots . . & \ldots . . & \ldots . . \\
d_{p-41} & d_{p-31} & d_{p-21} & d_{p-11} & \ldots . . & d_{p n}-\lambda
\end{array}\right|=0,
$$
by formulas (12).

It was established in [15] that the side coefficients of the determinant (13) have little effect on the frequency characteristics of the pipeline, the discrepancy in the frequencies of free vibrations is not more than $1 \%$. Due to small discrepancies in the results, to simplify the calculation, we neglect the coefficients $d_{m, m \pm 1}, d_{m, m \pm 2}$ in the future, taking them equal to zero. For a simplified diagonal determinant, the characteristic equation takes the form:

$$
\left(A_{1,1}-B_{1, n} \omega_{1 n}^{2}\right) \cdot\left(A_{2,1}-B_{1, n} \omega_{2 n}^{2}\right) \ldots\left(A_{m, n}-B_{m, n} \omega_{m n}^{2}\right)=0
$$

the roots of this equation are the squares of the frequencies of the natural vibrations of the cylindrical shell

$$
\omega_{m n}^{2}=\frac{A_{m, n}}{B_{m, n}}
$$

where $A_{m, n}$ and $B_{m, n}$ are defined (12).

Substituting in (15) instead of $A_{m, n}, B_{m, n}$ expressions from (12) we obtain the formula in general form for determining the frequency of natural oscillations:

$$
\omega_{m n}=\sqrt{\frac{\lambda_{n}^{4}+m^{4}\left(m^{2}-1\right)\left(m^{2}-1+p^{*}-2 q_{s l}^{*}\right)+\kappa^{*} m^{4}-\lambda_{n}^{4} m^{4} P / n^{2}}{\rho^{*} R h\left(\lambda^{2} h_{v}+m^{2}+m^{4}\right)+\mu_{b j} m^{4}+\rho_{0} \Phi_{m n} R^{2} m^{4}}}
$$

To obtain an expression for determining the frequency of natural vibrations with a nondeformable contour (rod theory), in (16) we substitute $m=1, n=1$. The expression takes the form: 


$$
\omega_{11}=\sqrt{\frac{\lambda_{n}^{4}+\kappa^{*}-\lambda_{n}^{4} P}{\rho^{*} R h\left(\lambda^{2} h_{v}+2\right)+\mu_{b j}+\rho_{0} \Phi_{m n} R^{2}}}
$$

To obtain an expression for determining the frequency of natural vibrations, taking into account the deformation of the cross section (shell theory), we substitute $m=2, n=1$ in (16). The expression takes the form:

$$
\omega_{21}=\sqrt{\frac{\lambda_{n}^{4}+48\left(3+p^{*}-2 q_{s l}^{*}\right)+16 \kappa^{*}-16 \lambda_{n}^{4} P}{\rho^{*} R h\left(\lambda^{2} h_{v}+20\right)+16\left(\mu_{b j}+\rho_{0} \Phi_{m n} R^{2}\right.}}
$$

Further, using the dynamic stability criterion, for which the frequency of free vibrations vanishes, from expression (16), given that the denominator is not equal to zero, we obtain:

$$
\lambda_{n}^{4}+m^{4}\left(m^{2}-1\right)\left(m^{2}-1+p^{*}-2 q_{s l}^{*}\right)+\kappa^{*} m^{4}-\lambda_{n}^{4} m^{4} P / n^{2}=0
$$

dividing each term of expression (19) by $m^{4}\left(m^{2}-1\right)$ we express the quantity:

$$
2 q_{s l}^{*}=m^{2}-1+p^{*}+\frac{\kappa^{*}}{\left(m^{2}-1\right)}+\frac{\lambda_{n}^{4}}{m^{4}\left(m^{2}-1\right)}-\frac{\lambda_{n}^{4} P}{n^{2}\left(m^{2}-1\right)}
$$

and substituting $\lambda_{n}=\frac{n \pi R}{L \sqrt{h_{v}}} ; p^{*}=p_{0} \frac{R}{E h \cdot h_{v}^{2}} ; \kappa^{*}=\frac{R^{2} \kappa}{E h \cdot h_{v}^{2}} ; q_{s l}^{*}=\frac{\gamma H R}{E h \cdot h_{v}^{2}} ; n=1$, we have:

$$
2 \gamma H \frac{R}{E h h_{v}^{2}}=\left(m^{2}-1\right)+p_{0} \frac{R}{E h h_{v}^{2}}+\frac{\kappa R}{\left(m^{2}-1\right)} \frac{R}{E h h_{v}^{2}}+\frac{\frac{n^{4} \pi^{4} R^{4}}{L^{4} h_{v}^{2}}}{m^{4}\left(m^{2}-1\right)}-\frac{\frac{n^{4} \pi^{4} R^{4}}{L^{4} h_{v}^{2}} P}{\left(m^{2}-1\right)},
$$

After mathematical transformations, we obtain the formula for determining the critical external pressure $P_{c r}=2 \gamma H$ on the oil pipeline:

$$
P_{c r}=\frac{\left(m^{2}-1\right) D}{R^{3}}+\frac{\kappa R}{m^{2}-1}+p_{0}+\frac{\pi^{4} R^{3} E h}{L^{4}\left(m^{2}-1\right)}\left(\frac{1}{m^{4}}-P\right)
$$

\[ D=\frac{E h^{3}}{12\left(1-v^{2}\right)} \text { is the cylindrical stiffness of the p } \]
where
At zero internal working pressure, for infinitely long sect
pression (22) acquires the critical external pressure formula
ing in an elastic medium that resists wall displacements:
\[ P_{c r}=\frac{\left(m^{2}-1\right) D}{R^{3}}+\frac{\kappa R}{m^{2}-1} \]

If the possibility of longitudinal deformations of the pipe is excluded, then the factor (1$v^{2}$ ), where $v$ is the Poisson's ratio of the pipe material, should be entered in the denominator of the first term of formula (21). Formula (23) for $m=2$ with $k=0$ turns into the well-known formula of M. Levy:

$$
P_{c r}=\frac{3 D}{R^{3}}
$$

These facts allow us to conclude that the approach to determining the critical external pressure is correct.

The resulting expression (22) is the most complete for determining the critical external pressure on the walls of the pipeline laid in the ground and allows you to take into account the geometric characteristics of the cross section, the coefficient of elastic resistance of the soil, the value of the longitudinal compressive force, the internal working pressure, as well as the length of the pipeline section. 


\section{Analysis of the data}

Using formula (16), we analyze the influence of the length parameter of the considered section $l^{*}=L / R$ on the frequency of free vibrations of the underground oil pipeline in various soil conditions (the elastic resistance coefficient of the soil is taken to be successively equal to $\kappa=0.05 \cdot 10^{7} \mathrm{~N} / \mathrm{m}^{3}, \kappa=0.8 \cdot 10^{7} \mathrm{~N} / \mathrm{m}^{3}, \kappa=1.2 \cdot 10^{7} \mathrm{~N} / \mathrm{m}^{3}$ ). We take the value of the internal working pressure equal to $p_{0}=1.8(\mathrm{MPa})$, the fluid flow velocity $V=$ $3.0 \mathrm{~m} / \mathrm{s}$, the depth of the pipeline $H=2 \mathrm{~m}$, the value of the longitudinal compressive force parameter we take $P=0$ and $P=0.2$. The data obtained are shown in Table 1 and 2 .

Table 1. Frequency of natural vibrations of oil pipelines with a laying depth of $\mathrm{H}=2.0 \mathrm{~m}$, wall thickness $h=R / 40$ in the absence of longitudinal force

\begin{tabular}{|c|c|c|c|c|c|c|c|c|c|c|}
\hline \multirow{3}{*}{$\begin{array}{c}\text { Freque } \\
\text { ncy } \\
\mathrm{Hz}\end{array}$} & \multicolumn{5}{|c|}{$\begin{array}{c}\kappa=0.05 \cdot 10^{7} \mathrm{~N} / \mathrm{m}^{3} ; p_{0}=0(\mathrm{mPa}) \\
V=3.0 \mathrm{~m} / \mathrm{s}\end{array}$} & \multicolumn{5}{|c|}{$\begin{array}{c}\kappa=0.05 \cdot 10^{7} \mathrm{~N} / \mathrm{m}^{3} ; p_{0}=1.8(\mathrm{mPa}) ; \\
V=3.0 \mathrm{~m} / \mathrm{s}\end{array}$} \\
\hline & \multicolumn{5}{|c|}{$\mathrm{L} / \mathrm{R}$} & \multicolumn{5}{|c|}{$\mathrm{L} / \mathrm{R}$} \\
\hline & 10 & 15 & 20 & 30 & 40 & 10 & 15 & 20 & 30 & 40 \\
\hline$\omega_{1.1}$ & 44.68 & 19.83 & 11.19 & 5.08 & 3.03 & 44.54 & 19.83 & 11.19 & 5.08 & 3.02 \\
\hline$\omega_{2.1}$ & 20.86 & 15.09 & 14.03 & 13.62 & 13.54 & 28.39 & 24.93 & 24.30 & 24.06 & 24.02 \\
\hline \multirow[t]{2}{*}{$\omega_{3.1}$} & 41.35 & 40.42 & 40.31 & 40.27 & 40.26 & 54.28 & 53.82 & 53.73 & 53.69 & 53.68 \\
\hline & \multicolumn{5}{|c|}{$\begin{array}{c}\kappa=0.8 \cdot 10^{7} \mathrm{~N} / \mathrm{m}^{3} ; p_{0}=0(\mathrm{mPa}) \\
V=3.0 \mathrm{~m} / \mathrm{s}\end{array}$} & \multicolumn{5}{|c|}{$\begin{array}{c}\kappa=0.8 \cdot 10^{7} \mathrm{~N} / \mathrm{m}^{3} ; p_{0}=1.8(\mathrm{mPa}) \\
V=3.0 \mathrm{~m} / \mathrm{s}\end{array}$} \\
\hline$\omega_{1.1}$ & 44.78 & 20.35 & 12.09 & 6.84 & 5.49 & 44.78 & 20.35 & 12.10 & 6.84 & 5.49 \\
\hline$\omega_{2.1}$ & 21.23 & 16.33 & 15.35 & 14.97 & 14.91 & 29.06 & 25.70 & 25.08 & 24.85 & 24.81 \\
\hline \multirow[t]{2}{*}{$\omega_{3.1}$} & 41.57 & 40.99 & 40.89 & 40.84 & 40.83 & 54.71 & 54.25 & 54.16 & 54.12 & 54.11 \\
\hline & \multicolumn{5}{|c|}{$\begin{array}{c}\kappa=0.8 \cdot 10^{7} \mathrm{~N} / \mathrm{m}^{3} ; p_{0}=0(\mathrm{mPa}) \\
V=3.0 \mathrm{~m} / \mathrm{s}\end{array}$} & \multicolumn{5}{|c|}{$\begin{array}{c}\kappa=1.2 \cdot 10^{7} \mathrm{~N} / \mathrm{m}^{3} ; p_{0}=1.8(\mathrm{mPa}) \\
V=3.0 \mathrm{~m} / \mathrm{s}\end{array}$} \\
\hline$\omega_{1.1}$ & 44.90 & 20.63 & 12.55 & 7.62 & 6.43 & 44.90 & 20.63 & 12.55 & 7.62 & 6.43 \\
\hline$\omega_{2.1}$ & 21.71 & 16.95 & 16.01 & 15.65 & 15.59 & 29.42 & 26.10 & 25.49 & 25.26 & 25.22 \\
\hline$\omega_{3,1}$ & 41.87 & 41.30 & 41.19 & 41.15 & 41.14 & 54.94 & 54.48 & 54.39 & 54.35 & 54.34 \\
\hline
\end{tabular}

Table 2. Frequency of natural vibrations of oil pipelines with a laying depth of $H=2.0 \mathrm{~m}$, wall thickness $h=R / 40$, and longitudinal force parameter $P=0.2$

\begin{tabular}{|c|c|c|c|c|c|c|c|c|c|c|}
\hline \multirow{3}{*}{$\begin{array}{c}\text { Freque } \\
\text { ncy } \\
\mathrm{Hz}\end{array}$} & \multicolumn{5}{|c|}{$\begin{array}{c}\kappa=0.05 \cdot 10^{7} \mathrm{~N} / \mathrm{m}^{3} ; p_{0}=0(\mathrm{mPa}) \\
V=3.0 \mathrm{~m} / \mathrm{s}\end{array}$} & \multicolumn{5}{|c|}{$\begin{array}{c}\kappa=0.05 \cdot 10^{7} \mathrm{~N} / \mathrm{m}^{3} ; p_{0}=1.8(\mathrm{mPa}) \\
V=3.0 \mathrm{~m} / \mathrm{s}\end{array}$} \\
\hline & \multicolumn{5}{|c|}{$\mathrm{L} / \mathrm{R}$} & \multicolumn{5}{|c|}{$\mathrm{L} / \mathrm{R}$} \\
\hline & 10 & 15 & 20 & 30 & 40 & 10 & 15 & 20 & 30 & 40 \\
\hline$\omega_{1.1}$ & 39.84 & 17.74 & 10.03 & 4.58 & 2.75 & 39.84 & 17.74 & 10.03 & 4.58 & 2.75 \\
\hline$\omega_{2.1}$ & - & 9.13 & 12.29 & 13.28 & 13.44 & 8.56 & 21.84 & 23.34 & 23.87 & 23.96 \\
\hline \multirow[t]{2}{*}{$\omega_{3.1}$} & 28.27 & 38.21 & 39.63 & 40.13 & 40.22 & 45.43 & 52.18 & 53.21 & 53.59 & 53.64 \\
\hline & \multicolumn{5}{|c|}{$\begin{array}{c}\kappa=0.8 \cdot 10^{7} \mathrm{~N} / \mathrm{m}^{3} ; p_{0}=0(\mathrm{mPa}) \\
V=3.0 \mathrm{~m} / \mathrm{s}\end{array}$} & \multicolumn{5}{|c|}{$\begin{array}{c}\kappa=0.8 \cdot 10^{7} \mathrm{~N} / \mathrm{m}^{3} ; p_{0}=1.8(\mathrm{mPa}) \\
V=3.0 \mathrm{~m} / \mathrm{s}\end{array}$} \\
\hline$\omega_{1.1}$ & 40.10 & 18.33 & 11.02 & 6.48 & 5.34 & 40.10 & 18.33 & 11.02 & 6.48 & 5.34 \\
\hline$\omega_{2.1}$ & - & 11.05 & 13.78 & 14.67 & 14.81 & 10.59 & 22.71 & 24.15 & 24.67 & 24.75 \\
\hline \multirow[t]{2}{*}{$\omega_{3.1}$} & 29.08 & 38.82 & 40.21 & 40.71 & 40.79 & 45.95 & 53.62 & 53.65 & 54.02 & 54.08 \\
\hline & \multicolumn{5}{|c|}{$\begin{array}{c}\kappa=0.8 \cdot 10^{7} \mathrm{~N} / \mathrm{m}^{3} ; p_{0}=0(\mathrm{mPa}) \\
V=3.0 \mathrm{~m} / \mathrm{s}\end{array}$} & \multicolumn{5}{|c|}{$\begin{array}{c}\kappa=1.2 \cdot 10^{7} \mathrm{~N} / \mathrm{m}^{3} ; p_{0}=1.8(\mathrm{mPa}) \\
V=3.0 \mathrm{~m} / \mathrm{s}\end{array}$} \\
\hline$\omega_{1.1}$ & 40.24 & 18.63 & 11.52 & 7.29 & 6.30 & 40.24 & 18.63 & 11.52 & 7.29 & 6.30 \\
\hline$\omega_{2.1}$ & - & 11.95 & 14.52 & 15.36 & 15.49 & 11.52 & 23.16 & 24.58 & 25.08 & 25.17 \\
\hline$\omega_{3.1}$ & 29.51 & 39.14 & 40.52 & 41.02 & 41.10 & 46.22 & 52.86 & 53.88 & 54.25 & 54.31 \\
\hline
\end{tabular}


a)

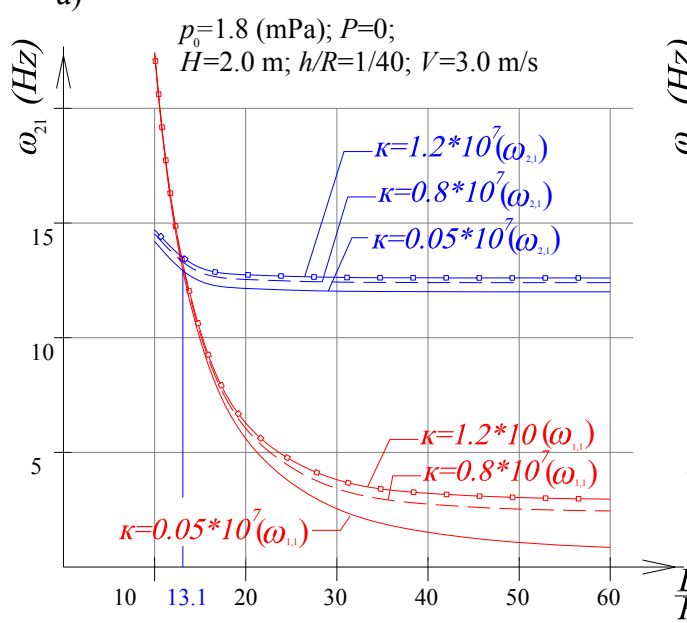

b)

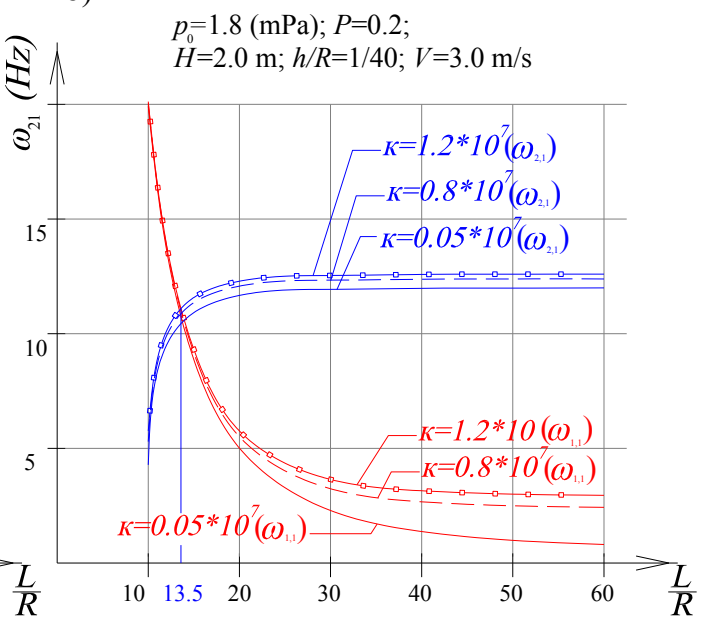

Fig. 2. Graphs of the dependence of the frequency of free vibrations for $m=1$ (red) and $m=2$ (blue) on the length parameter of the pipeline section for pressureless pipes (a) in the absence of longitudinal force; b) the longitudinal force parameter is 0.2 )

a)

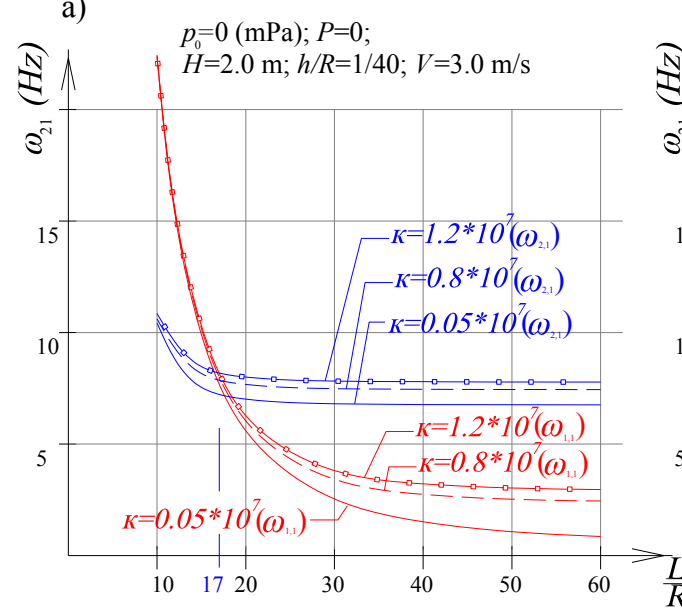

b)

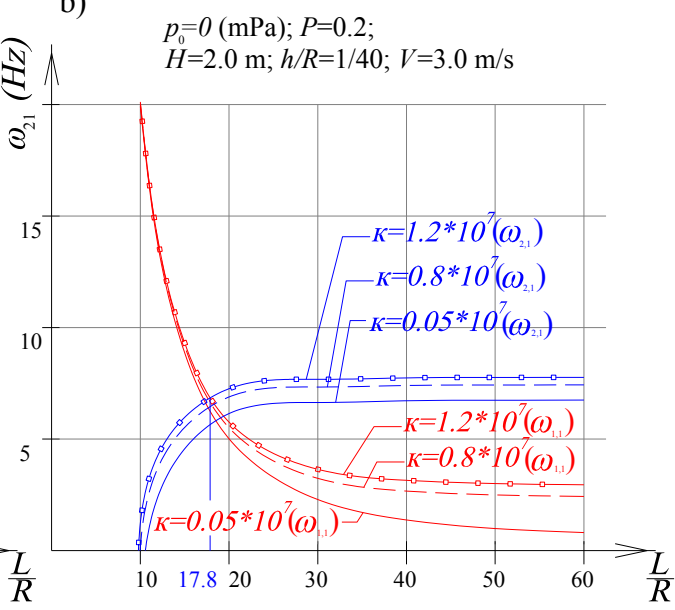

Fig. 3. Graphs of the dependence of the frequency of free vibrations for $m=1$ (red) and $m=2$ (blue) on the length parameter of the pipeline section at internal pressure $p_{0}=1.8 \mathrm{MPa}(\mathrm{a})$ in the absence of longitudinal force; $b$ ) the longitudinal force parameter is 0.2 )

Based on the data presented in Tables 1 and 2, as well as in Figure 1, we can draw the following conclusions:

1. The minimum frequencies for the value of the pipeline length parameter of less than 13.1 were obtained for $m=2, n=1$, which corresponds to shell modes of vibration, for $L / R$ greater than 13.1, the frequency of free vibrations is realized according to the rod theory $(m=2, n=1)$;

2.In the absence of longitudinal compressive force with increasing length of the pipeline section, the frequency $\omega_{11}$ and $\omega_{21}$ decreases, and for pipelines under the influence of the longitudinal compressive force, the frequencies $\omega_{21}$ (corresponding to shell modes) increase, as can be seen in the graphs (Fig. 1); 
3. The frequency of free vibrations obtained according to the rod theory $\omega_{11}$ decreases sharply with increasing length parameter, for example, from the data in Table 1 for $l^{*}=L / R=10$ for $\kappa=1.2 \cdot 10^{7} \mathrm{~N} / \mathrm{m}^{3} \omega_{11}=44.9 \mathrm{~Hz}$, and for $l^{*}=L / R=60 \omega_{11}=5.92 \mathrm{~Hz}$. The decrease in frequency occurs 7.5 times.

4. The longitudinal force parameter significantly affects the frequencies of free vibrations for the length of the pipeline section less than $L=13.5 R$. When the length of the section decreases, the frequency of free vibrations drops sharply.

5. From the analysis of the graphs shown in Figure 1, it can be seen that for some values of the parameter $l^{*}=L / R$, the minimum frequencies for the rod and shell theory coincide, $\omega_{11}=\omega_{21}$. Based on this fact, we can conclude that the parameter $l *$ sets the criterion for the applicability of the shell and rod theory. Equating expressions (17) and (18) with each other and using mathematical transformations, we obtain the expression of the critical parameter of the pipeline length $l^{*}$ :

$$
l^{*}=\frac{L}{R}=\frac{\pi}{\sqrt{h_{v}}} \sqrt[4]{\frac{\rho^{*} R h(18+12 P)+15 \mu_{b j}}{12 \kappa^{*} \rho^{*} R h+48\left(3+\rho^{*}-2 q_{s l}^{*} R h+\mu_{b j}^{*}\right)}}
$$

in the absence of internal working pressure and longitudinal compressive force, expression (26) takes the form:

$$
l^{*}=\frac{L}{R}=\frac{\pi}{\sqrt{h_{v}}} \sqrt[4]{\frac{18 \rho^{*} R h+15 \mu_{b j}}{12 \kappa^{*} \rho^{*} R h+48\left(3-2 q_{s l}^{*} R h+\mu_{b j}^{*}\right)}}
$$

Analyzing expressions (25) and (26), we can draw the following conclusions:

a) the internal working pressure has a significant effect on the value of $l^{*}$. With increasing parameter $p^{*}$, the length parameter $l^{*}=L / R$ decreases, because the internal working pressure prevents the deformation of the cross section of the pipeline, bringing the design scheme closer to a rod with an undeformable cross-section contour (see Fig. 1, 2). This is confirmed by the calculated data of Tables 1 and 2, for example, for the coefficient of elastic resistance of the soil $\kappa=0.8 \cdot 10^{7} \mathrm{~N} / \mathrm{m}^{3}$, for the length of the section $L=10 R$ in the absence of internal pressure, the frequency of free vibrations is $\omega_{21}=21.23 \mathrm{~Hz}$, and at pressure $p_{0}=1.8 \mathrm{mPa} \omega_{21}=29.06 \mathrm{~Hz}$ (see Table 1).

b) as the elastic rebound parameter $\kappa^{*}$ increases, the length $l^{*}$ decreases, for example, $p_{0}=0$ and $P=0.2$ for $\kappa=0.05 \cdot 10^{7}\left(\mathrm{~N} / \mathrm{m}^{3}\right) l^{*}=L / R=13.1$, and for $\kappa=1.2 \cdot 10^{7}\left(\mathrm{~N} / \mathrm{m}^{3}\right)$ ceteris paribus length parameter $l^{*}=L / R=12.93$ (see Fig. 1);

c) for $l \leq l^{*}$, frequencies should be determined according to (18), for $l \geq l^{*}$ the frequencies are determined according to (17) that is, according to the rod theory for a nondeformable cross-section contour;

d) with increasing depth of the pipeline and soil pressure $q^{*}$ sl, the value of the length parameter $l^{*}$ increases, that is, the applicability boundary shifts toward the theory of shells;

e) with an increase in the added mass of soil, the length $l^{*}$ decreases;

f) the longitudinal compressive force parameter P has little effect on the length $l^{*}$ since it reduces the frequencies of free vibrations not only for $m=2, n=1$ (according to the theory of shells [1-10]), but also for $m=1, n=1$ (according to the theory of rods [11, 12]).

\section{References}

1. E. Ruocco, L. Di, Raffaele, V. Minutolo, Procedia Earth and Planetary Science, 16, 2534 (2016). DOI: 10.1016/j.proeps.2016.10.004 
2. F. Dong, X. Bie, J. Tian, X. Xie and G. F. Du, Experimental and Numerical Study on the Strain Behavior of Buried Pipelines Subjected to an Impact Load. (2019). DOI: 10.3390/app9163284

3. A. Massa, N. Galgoul, N. Junior, A. Fernandes, F. Coelho, S. Neto, The Influence of Internal Pressure on Pipeline Natural Frequency (2009). DOI: 10.1115 / OMAE200979666

4. O. Fyrileiv, O. Aamlid, E. Andreassen, Pipeline Free Spans: Influence of Internal Pressure (2010). DOI: 10.1115 / OMAE2010-20628

5. S. Georgiadou, E. Loukogeorgaki, D. Angelides, Proceedings of the International Offshore and Polar Engineering Conference, 80-87 (2014). https://www.onepetro.org/conference-paper/ISOPE-I-14-275

6. W.-H. Xü, W.-D. Xie, X.-F. Gao, Y.-X. Ma, Journal of Ship Mechanics, 22, 446-453 (2018) DOI: 10.3969/j.issn.1007-7294.2018.04.007

7. G. P. Kourctzis, Sloan, Analysis of buried pipelines subjected to ground surface settlement and heave. (2014) DOI: 10.1139/cgj-2014-0332

8. V. Sokolov, I. Razov and Y. Ogorodnova, Effect of Internal Pressure on Parametric Vibrations and Dynamic Stability of Thin-Walled Ground Pipeline Larger Diameter Connect with Elastic Foundation (2016). DOI: 10.1051/matecconf/20167304002

9. V. Sokolov, I. Razov, Free vibrations of thin-walled semi-underground main gas pipelines of large diameter, with the attached ground mass. (2017) DOI: 10.1051/matecconf/201710602021

10. S. Bochkarev, Computational Continuum Mechanics, 10, 406-415. (2017). DOI: 10.7242/1999-6691/2017.10.4.32.

11. E.L. Kuzneczova, D.V. Leonenko, E`.I. Starovojtov, MTT, 3, 152-160 (2015). DOI: $10.3103 / \mathrm{S} 0025654415030127$

12. J. Dražić, N. Vatin. Procedia Engineering. 2016. 165. Pp. 883-890. DOI:10.1016/j.proeng.2016.11.788.

13. G.G. Sheng, X. Wang, J. Reinf. Plast. Comp., 27(2), 117-134 (2008). DOI: 10.5050/KSNVE.2013.24.3.261

14. L.H. Donnel, Trans. ASME, 56(11), 86-94 (1934). https://studylib.net/doc/8786021/anew-theory-for-the-buckling-of-thin-cylinders-under-axial

15. A.V. Dmitriev, V.G. Sokolov, A.V. Bereznyov Transportny`e sooruzheniya, 3, (2019) https://t-s.today/PDF/40SATS319.pdf 